\title{
Herramienta de sondeos operativa en AEMET
}

https://doi.org/10.31978/639-19-010-0.193

\author{
Álvaro Subías Díaz-Blanco'1 (asubiasd@aemet.es) \\ Alberto Fernández Matía ${ }^{1}$ (afernandezm@aemet.es) \\ Alejandro Roa Alonso ${ }^{1}$ (aroaa@aemet.es) \\ Marcos Natanael Gómez Molina² (mgomezm@aemet.es)
}

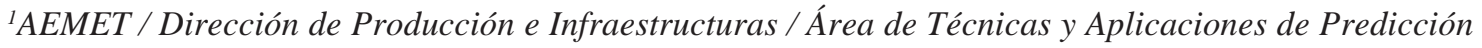

${ }^{2}$ AEMET / Delegación Territorial en Cantabria

\begin{abstract}
RESUMEN
En AEMET se ha desarrollado la herramienta de visualización de sondeos SkewT debido a la necesidad de representación a partir del formato estándar binario bufr. En el presente artículo mostramos las distintas posibilidades que ofrece esta herramienta de visualización que se ha convertido en la herramienta de referencia, además de su uso original, para representación de sondeos previstos, de hodógrafas, y en general de aquellas variables relativas a modelos numéricos relacionadas con la vertical de un punto.
\end{abstract}

PALABRAS CLAVE: radiosondeos.

\section{INTRODUCCIÓN}

SkewT es una herramienta desarrollada en el Área de Técnicas y Aplicaciones de Predicción de AEMET. Se ha desarrollado en Python 2 utilizando la librería de dibujo matplotlib, que se distribuye dentro de la versión de Python, y la librería eccodes de lectura y escritura de formatos bufr y grib desarrollada en ECMWF.

Al haber sido desarrollada íntegramente en AEMET se consigue una gran flexibilidad para las necesidades planteadas por distintos usuarios. Los cálculos termodinámicos no hacen uso de librerías externas sino que han sido escritos en una clase dentro del código.

Con anterioridad en AEMET la herramienta de sondeos operativa había sido ysond programada en McIdas. La nueva herramienta, al igual que ysond, permite la visualización de sondeos observados y previstos.

\section{SONDEOS OBSERVADOS}

Diariamente se dibujan sondeos de España y Portugal en el Área de Explotación y Gestión de Datos. Se leen ficheros en formato bufr generados por radiosondeos que toman muestras cada 2 segundos (en torno a 2000 niveles verticales). Los sondeos dibujados con SkewT se guardan en el archivo desde el 24 de junio de 2016. También están disponibles en AEMET bufr de radiosondeos de todo el mundo.

\begin{tabular}{|l|l|}
\hline 08001 & Coruña \\
\hline 08023 & Santander \\
\hline 08190 & Barcelona \\
\hline 08221 & Madrid \\
\hline 08302 & Palma de Mallorca \\
\hline 08430 & Murcia \\
\hline 06018 & Tenerife \\
\hline
\end{tabular}

Tabla 1. Lista de radiosondeos de España, lanzados a las 00 y 12 UTC. 
Además de las curvas habituales de temperatura y temperatura de rocío, en el diagrama se representan la temperatura equivalente y la temperatura del termómetro húmedo. Se ha añadido la temperatura de fase, introducida por Alberto Fernández (AEMET) para el cálculo de la cota de nieve (véanse los detalles en el artículo «La temperatura de fase en la transición de nieve a lluvia: definición y aplicación al

\begin{tabular}{|l|l|}
\hline 08577 & Lisboa \\
\hline 08508 & Lajes-Azores \\
\hline 08522 & Funchal-Madeira \\
\hline
\end{tabular}

Tabla 2. Lista de radiosondeos de Portugal, lanzados a las 12 UTC. cálculo de la cota de nieve»).
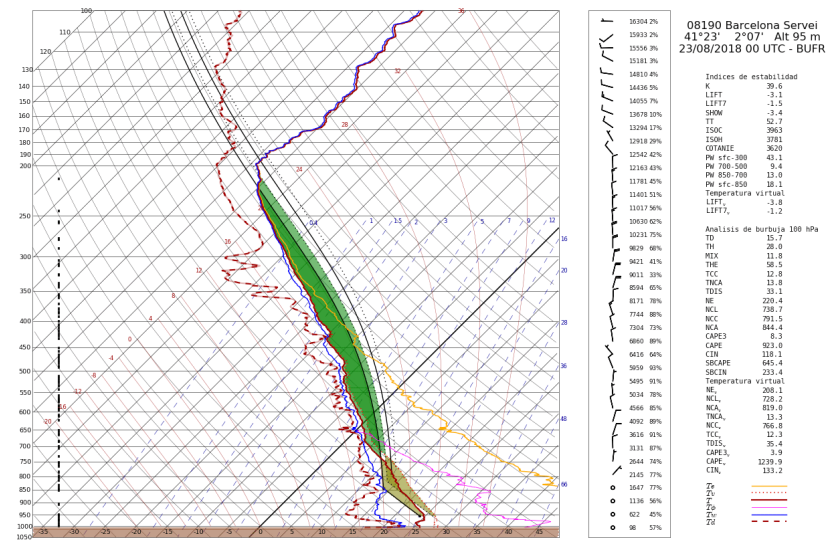

Figura 1. Sondeo observado de Barcelona el 23 de agosto de 2018 a las 00 UTC.
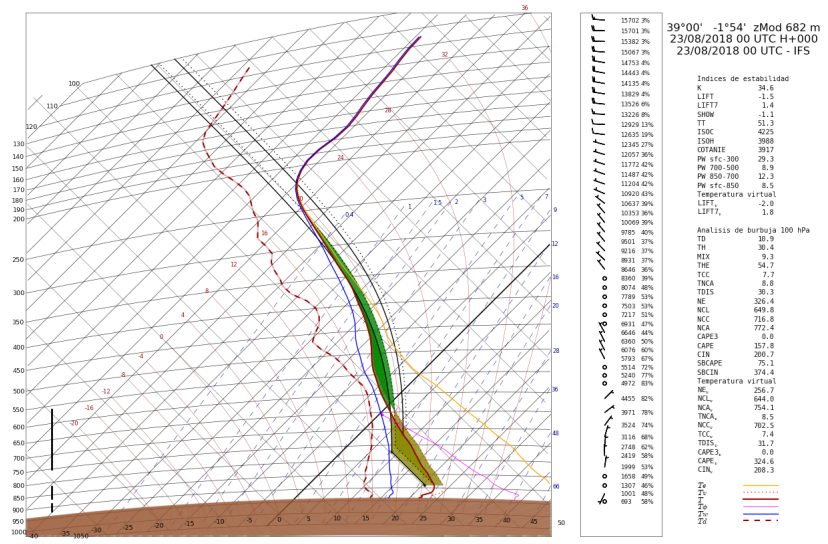

Figura 2. Representación de un sondeo mediante un tefigrama $(T, \phi)$.

Los sondeos pueden ser representados tanto en un diagrama oblicuo $T$ - $\log p$ como en un tefigrama. Esto se consigue al realizar un dibujo en dos pasos de tiempo, uno en el que se establecen coordenadas $(T, p)$ inherentes a los datos termodinámicos, y otro en el que se hace una conversión de estas coordenadas a las coordenadas de la pantalla $(x, y)$, operación que depende del tipo de diagrama aerológico elegido.

\section{SONDEOS PREVISTOS}

A partir del programa inicial que representa sondeos observados se ha añadido la posibilidad de que la lectura de datos pudiese provenir de otra fuente. Si se desea la representación de sondeos previstos se trataría de obtener los datos a partir de la vertical de cualquier punto de rejilla de un fichero grib. Los diagramas aerológicos son generados a partir de todos los niveles híbridos de los modelos HARMONIEAROME e IFS (ECMWF).
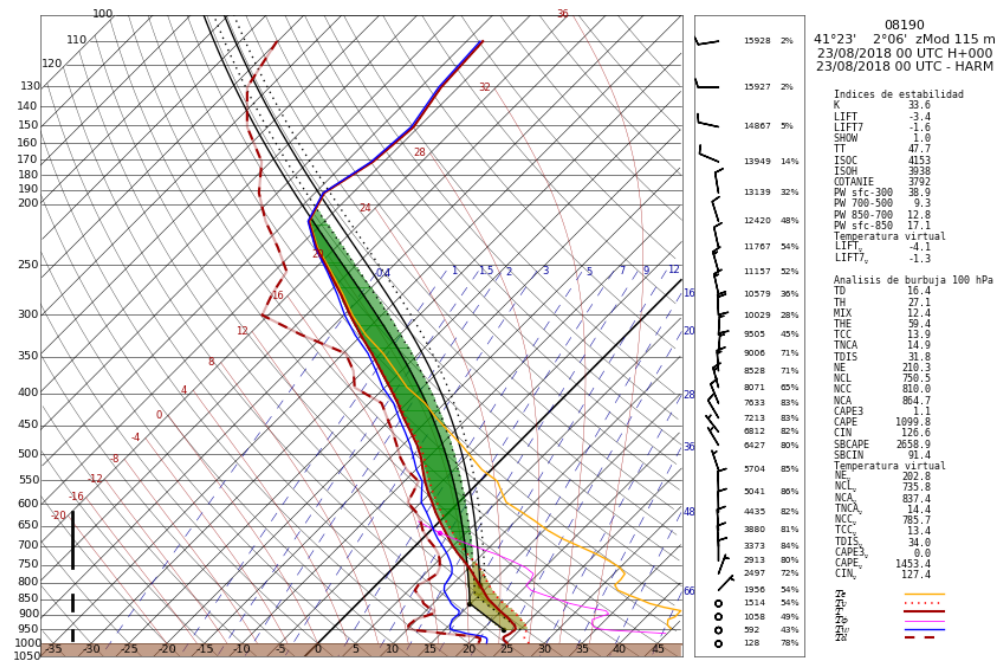

Figura 3.

Sondeo previsto de

HARMONIE-AROME.

El punto de rejilla se ha escogido como el más próximo al lugar de lanzamiento de radiosondeo de Barcelona. 


\section{HODÓGRAFA}

Se ha añadido la opción de representación de la hodógrafa asociada al sondeo al poder disponer de los datos de viento en altura. Representamos los vientos en nudos, junto con el viento medio calculado con el método de Bunkers, también se indica la posible dirección de tormentas a izquierda y a derecha (splitting storm).

En cuanto a postproceso de índices se calcula la cizalladura entre distintos niveles, el módulo del viento medio así como la helicidad relativa a la tormenta y otros índices asociados (Bulk Richardson Number, EHI...). Se considera además en los cálculos la capa efectiva, que es aquella en la que la burbuja que asciende adiabáticamente tiene valores de energía potencial convectiva por ascenso adiabático (CAPE) superior a $100 \mathrm{~J} / \mathrm{kg}$ y de inhibición convectiva (CIN) inferior a $250 \mathrm{~J} / \mathrm{kg}$.

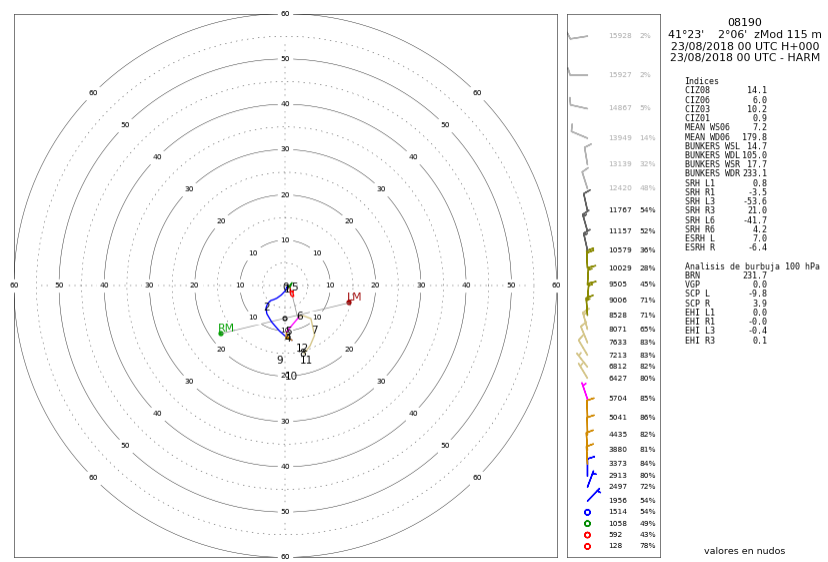

Figura 4. Hodógrafa asociada al sondeo previsto de la figura 3.

\section{TABLAS}

Junto con las salidas gráficas se adjunta un listado de índices asociados al sondeo, clasificados en índices de estabilidad y variables del sondeo, índices asociados a la cizalladura del viento e índices relativos al análisis de burbuja. Gráficamente se muestra por defecto una burbuja representativa de una capa con un espesor $100 \mathrm{hPa}$. En las tablas se consideran distintos espesores así como una burbuja que parte desde la superficie. Los cálculos se hacen tanto con temperatura como con temperatura virtual, este último más correcto para el cálculo del CAPE debido a que el cálculo de la flotabilidad necesita la contribución de la humedad del aire. Para la ejecución del programa estos valores son configurables.

A su vez se incluye una tabla con los valores utilizados por el programa en los cálculos. Las filas representan los niveles y las columnas las variables consideradas, algunas de las cuales son leídas directamente de los ficheros de entrada bufr, grib o ascii y otras son calculadas a partir de las primeras.

Figura 5.

Fichero de salida de texto mostrando algunos de los índices calculados.

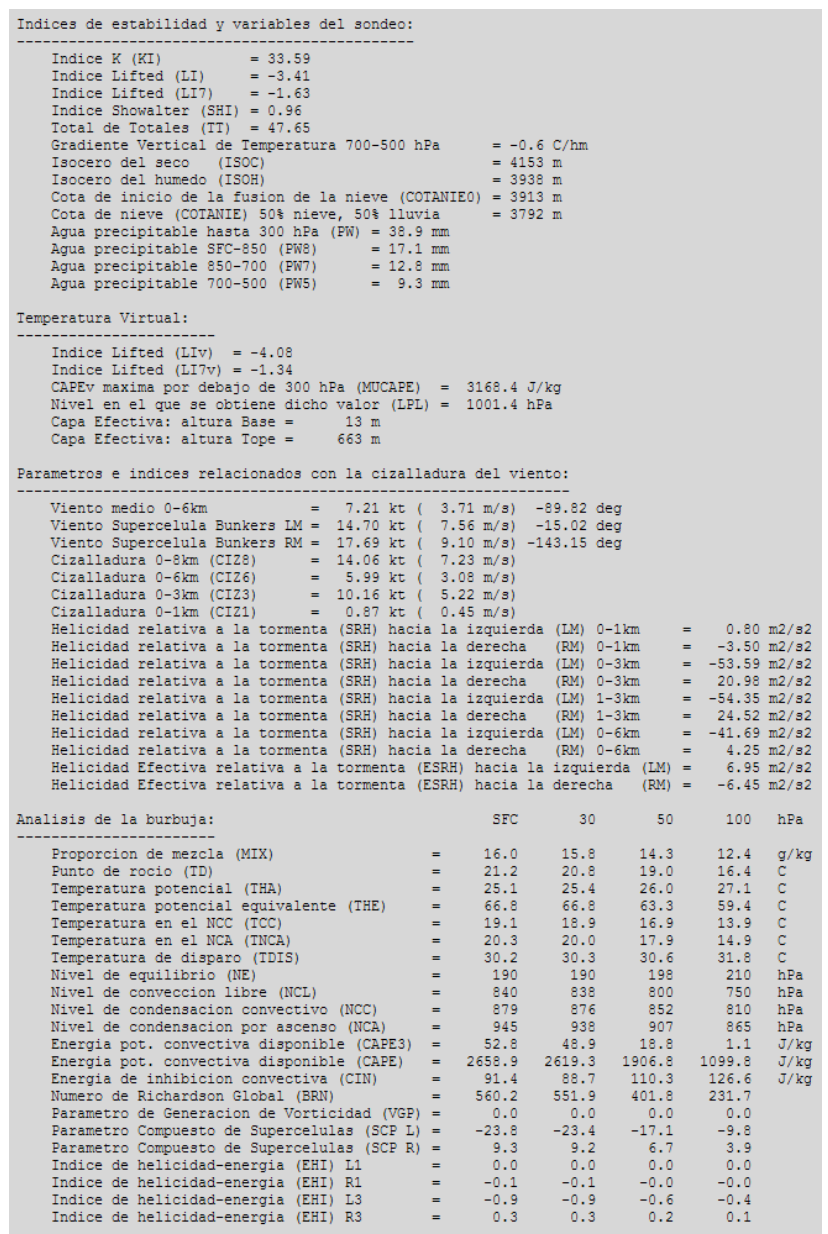




\section{COMPARACIÓN ENTRE SONDEOS}

En la figura 6 se muestra la comparación del radiosondeo de Barcelona de 23/08/2018 de las 00 UTC junto con el análisis de HARMONIE-AROME. Esta herramienta nos permite ver la calidad de la predicción en la vertical de un punto además de hacernos una idea de la resolución vertical del modelo.

Puede ser útil la comparación entre modelos o entre alcances. También puede ser de utilidad para los desarrolladores del modelo como puede ser, por ejemplo, para poder evaluar en la vertical de un punto de rejilla un cambio en el método de integración vertical comparando branches de código (diferentes cambios en un sistema de control de versiones de código).
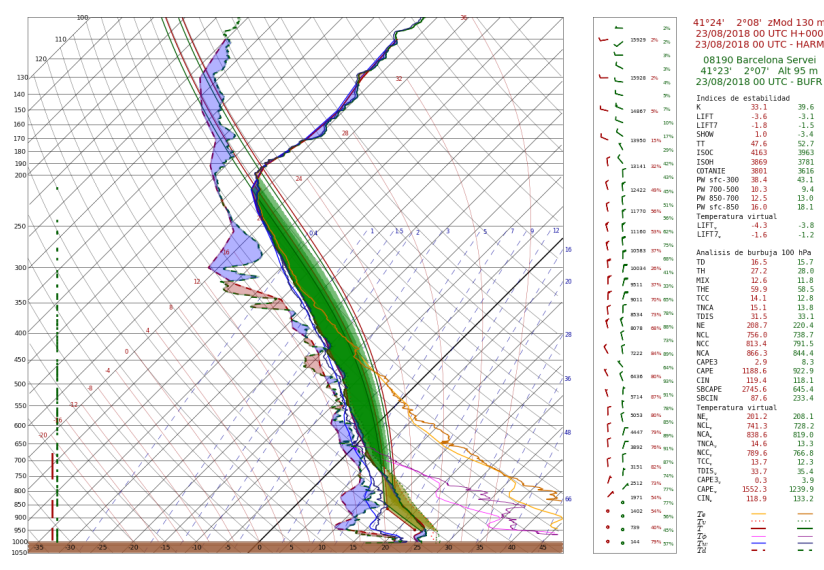

Figura 6. Comparación de un sondeo observado con el análisis HARMONIE-AROME.

\section{INTERFAZ WEB}

Se ha desarrollado una interfaz web que permite un uso sencillo de la aplicación para poder dibujar sondeos en puntos definidos por el usuario, debido a que SkewT funciona a través de línea de comandos. Se puede escoger entre modelos HARMONIEAROME (48 horas de alcance) e IFS (120 horas) estando disponible para dominios que cubren la península ibérica y las islas Canarias. La cantidad de puntos de rejilla en las salidas de los modelos actuales es muy grande por lo que solo se precalculan algunos de ellos, como es el caso de las localizaciones de aeropuertos o los lugares en los que se realiza lanzamiento de radiosondeos. El resto de sondeos se calculan a demanda.

Al seleccionar con el ratón en un punto del mapa se representa el sondeo previsto para el punto de rejilla más cercano para un alcance dado. La resolución es de 2,5 $\mathrm{km}$ en una rejilla regular en espacio Lambert para HARMONIE-AROME y de $0,1^{\circ}$ en una rejilla regular en latitud-longitud para IFS.

En la representación de sondeos previstos la orografía que se tiene en cuenta es la del propio modelo.

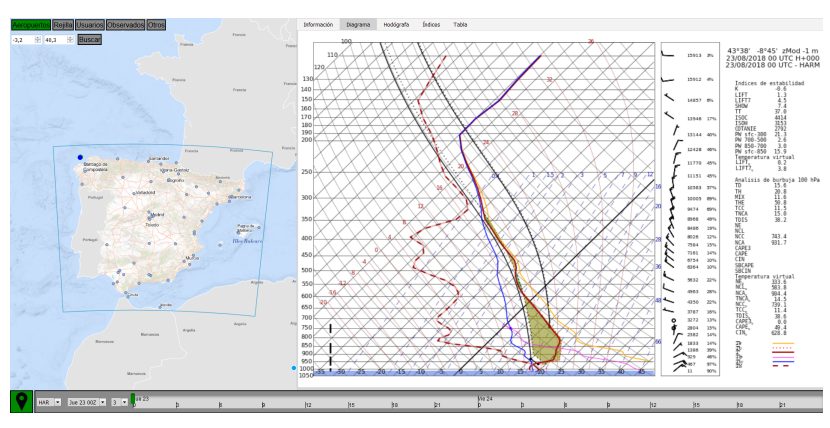

Figura 7. Interfaz web de acceso a sondeos observados y previstos (intranet de AEMET).

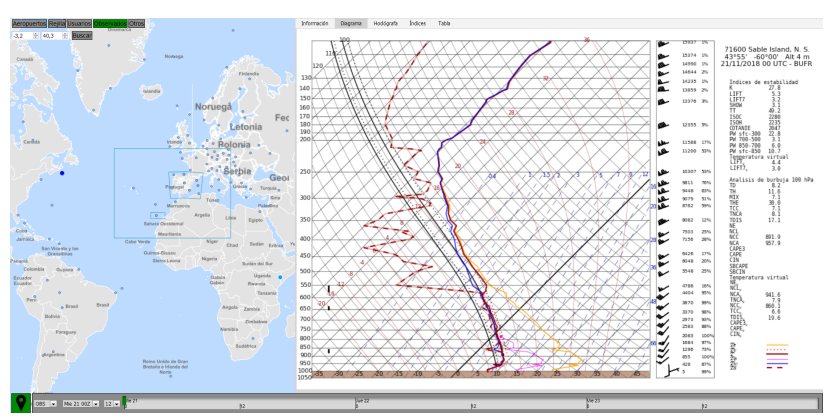

Figura 8. Radiosondeos del mundo.

En la imagen representamos el radiosondeo

lanzado desde la isla Sable (Canadá)

el 21 de noviembre de 2018 a las 00 UTC. Como ejemplo extremo mostramos en las figuras 9 y 10 sondeos en la cima del Teide calculados con HARMONIE-AROME e IFS respectivamente. Existen dos factores a tener en cuenta para la orografía:

- La orografía en los modelos está suavizada a su resolución espectral, resuelve tanto mejor cuanto mayor número de ondas se consideran.

- En la interfaz web se representa el punto de rejilla más próximo (punto verde), que es tanto más cercano al elegido cuanto mayor sea la resolución. 


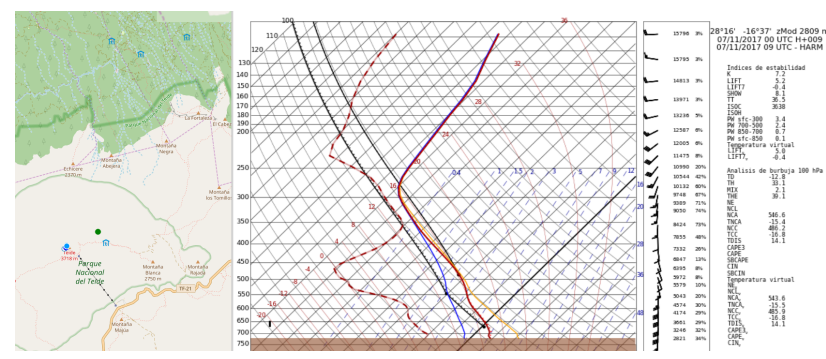

Figura 9. Sondeo previsto según el modelo

HARMONIE-AROME del punto de rejilla más próximo a la cima del Teide.

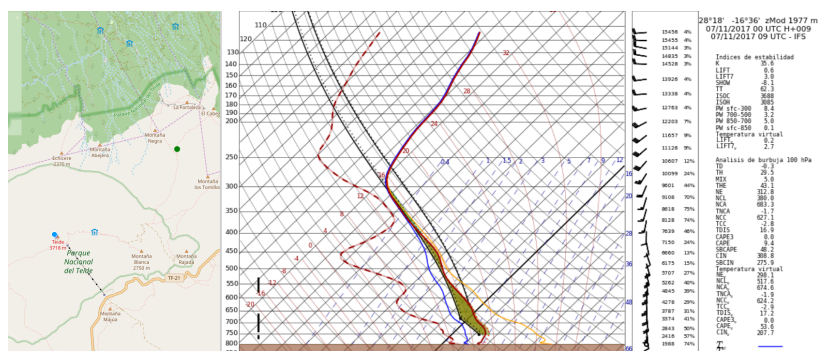

Figura 10. Sondeo previsto según el modelo IFS (ECMWF) del punto de rejilla más próximo a la cima del Teide.

Es importante fijarse que en el ejemplo del modelo IFS la altitud del sondeo está $900 \mathrm{~m}$ por debajo de HARMONIE-AROME.

\section{CONCLUSIONES}

El desarrollo de SkewT ha supuesto un modelo de desarrollo en el que a partir de una funcionalidad específica se ha creado un programa capaz de alcanzar un alto grado de versatilidad en la representación de diagramas aerológicos, convirtiéndose en una herramienta de visualización estándar en AEMET. Ello supone un paradigma de desarrollo para aquellas aplicaciones surgidas a partir de necesidades específicas de usuarios pero que puedan ser transpuestas a usos más generales.

\section{REFERENCIAS}

MarkowsKi, P. y Richarson, Y., 2010. Mesoscale Meteorology in Midlatitudes. Royal Meteorology Society.

Thompson, R. L., Edwards, R., Hart, J. A., Elmore, K. L. y Markowski, P., 2003. Close Proximity Soundings within Supercell Environments Obtained from the Rapid Update Cycle. Weather and Forecasting, 18 (6), 1243-1261.

StUll, R., 2011. Wet-bulb temperature from relative humidity and air temperature. Journal of Applied Meteorology and Climatology, 50 (11). 2267-2269.

DAVIES-Jones, R., 2007. An efficient and accurate method for computing the wet-bulb temperature along Pseudoadiabats. Monthly Weather Review, 136, 2764-2785.

Bunkers, M. J., Klimowski, B. A., Zeitler, J. W., Thompson, R. L. y Weisman, M. L., 2000. Predicting supercell motion using a new hodograph technique. Weather and Forecasting, 15, 61-79.

Alduchov, O. A. y Eskridge, R., 1996. Improved Magnus form approximation of saturation vapor pressure. Journal of Applied Meteorology, 35, 601-609.

García MÉndeZ, A., 1992. Temperatura potencial del termómetro húmedo: cálculo y aplicaciones. Nota técnica 5 STAP. Instituto Nacional de Meteorología.

Thompson, R. L., Mead, C. M. y Edwards, R., 2006. Effective storm-relative helicity and bulk shear in supercell thunderstorm environments. Weather and Forecasting, 22, 102-115.

Rasmussen, E. N. y Blanchard, D. O., 1998. A baseline climatology of sounding-derived supercell and tornado forecast parameters. Weather and Forecasting, 13, 1148-1164. 
